\title{
Distinct transcriptional MYCN/C-MYC activities are associated with spontaneous regression or malignant progression in neuroblastomas
}

\author{
Frank Westermann ${ }^{\star *}$, Daniel Muth ${ }^{\star *}$, Axel Benner ${ }^{\dagger}$, Tobias Bauer ${ }^{\ddagger}$, Kai- \\ Oliver Henrich ${ }^{*}$, André Oberthuer§, Benedikt Brors ${ }^{\ddagger}$, Tim Beissbarthף, \\ Jo Vandesompele ${ }^{\ddagger}$, Filip Pattyn ${ }^{\ddagger}$, Barbara Hero ${ }^{\S}$, Rainer König ${ }^{\ddagger}$, \\ Matthias Fischer§ and Manfred Schwab*
}

\begin{abstract}
Addresses: * Department of Tumor Genetics, German Cancer Research Center, Im Neuenheimer Feld 280, Heidelberg, 69120, Germany. ${ }^{\dagger}$ Department of Biostatistics, German Cancer Research Center, Im Neuenheimer Feld 280, Heidelberg, 69120, Germany. ${ }^{*}$ Theoretical Bioinformatics, German Cancer Research Center, Im Neuenheimer Feld 280, Heidelberg, 69120, Germany. \$Department of Pediatric Oncology, University Children's Hospital of Cologne, Kerpener Strasse 62, Cologne, 50924, Germany. "Division of Molecular Genome Analysis, German Cancer Research Center, Im Neuenheimer Feld 580, Heidelberg, 69120, Germany. ${ }^{¥}$ Center for Medical Genetics, Ghent University Hospital, De Pintelaan 185, Ghent, 9000, Belgium.
\end{abstract}

These authors contributed equally to this work.

Correspondence: Frank Westermann. Email: f.westermann@dkfz.de

Published: 13 October 2008

Genome Biology 2008, 9:RI50 (doi:10.1 I86/gb-2008-9-10-rI50)

The electronic version of this article is the complete one and can be found online at http://genomebiology.com/2008/9/I0/R/50
Received: 6 August 2008

Revised: 19 September 2008

Accepted: 13 October 2008

(c) 2008 Westermann et al.; licensee BioMed Central Ltd.

This is an open access article distributed under the terms of the Creative Commons Attribution License (http://creativecommons.org/licenses/by/2.0), which permits unrestricted use, distribution, and reproduction in any medium, provided the original work is properly cited.

\begin{abstract}
Background: Amplified MYCN oncogene resulting in deregulated MYCN transcriptional activity is observed in $20 \%$ of neuroblastomas and identifies a highly aggressive subtype. In MYCN single-copy neuroblastomas, elevated MYCN mRNA and protein levels are paradoxically associated with a more favorable clinical phenotype, including disseminated tumors that subsequently regress spontaneously (stage 4s-non-amplified). In this study, we asked whether distinct transcriptional MYCN or c-MYC activities are associated with specific neuroblastoma phenotypes.

Results: We defined a core set of direct MYCN/c-MYC target genes by applying gene expression profiling and chromatin immunoprecipitation (ChIP, ChIP-chip) in neuroblastoma cells that allow conditional regulation of MYCN and c-MYC. Their transcript levels were analyzed in $25 \mathrm{I}$ primary neuroblastomas. Compared to localized-nonamplified neuroblastomas, MYCN/c-MYC target gene expression gradually increases from stage 4s-non-amplified through stage 4-non-amplified to MYCN amplified tumors. This was associated with MYCN activation in stage 4snon-amplified and predominantly c-MYC activation in stage 4-non-amplified tumors. A defined set of MYCN/c-MYC target genes was induced in stage 4-non-amplified but not in stage 4s-non-amplified neuroblastomas. In line with this, high expression of a subset of MYCN/c-MYC target genes identifies a patient subtype with poor overall survival independent of the established risk markers amplified MYCN, disease stage, and age at diagnosis.

Conclusions: High MYCN/c-MYC target gene expression is a hallmark of malignant neuroblastoma progression, which is predominantly driven by c-MYC in stage 4-non-amplified tumors. In contrast, moderate MYCN function gain in stage 4s-non-amplified tumors induces only a restricted set of target genes that is still compatible with spontaneous regression.
\end{abstract}




\section{Background}

Neuroblastoma is the most common extracranial malignant solid tumor in early childhood. Clinical courses are highly variable, ranging from spontaneous regression to therapyresistant progression. Clinical and biological features, such as age at diagnosis, disease stage, numerical (ploidy) and structural chromosomal alterations (MYCN gene amplification; 1p, 3p, 11q deletions; 17q gain), are associated with patient outcome [1,2]. Amplified MYCN oncogene identifies a subtype with poor prognosis [3] and is consistently associated with high MYCN mRNA and protein levels. There is strong experimental evidence (ectopic MYCN expression in cell lines, N-myc transgenic neuroblastoma mouse model) that increased MYCN activity is involved in tumor initiation and progression of at least a subset of neuroblastomas $[4,5]$.

The $M Y C$ gene family members, c-MYC, MYCN and $M Y C L$, are involved in the biology of many cancer types. They encode basic helix-loop-helix leucine zipper proteins that are found as heterodimers with their obligate partner protein, MAX [6]. The MYC-MAX heterodimer binds to DNA consensus core binding sites, 5'-CACGTG-3' or variants thereof (E-boxes), which preferentially leads to transcriptional activation of target genes. Repression of target genes by MYC proteins has also been described [7]. This seems to be independent of the binding of MYC proteins to E-boxes, but involves a cofactor, Miz-1, that tethers MYC-MAX to gene promoters, such as p15 and $p 21$. Enhanced activity of MYC transcription factors contributes to almost every aspect of tumor formation: unrestricted cell proliferation, inhibition of differentiation, cell growth, angiogenesis, reduced cell adhesion, metastasis, and genomic instability [6,8]. In contrast, MYC transcription factors, including MYCN, also sensitize cells for apoptosis, a function that should inhibit tumor formation and that could also be involved in spontaneous tumor regression [9].

Spontaneous tumor regression does occur in neuroblastoma, at a higher frequency than in any other cancer type. This process resembles the physiological concurrence of massive cellular suicide (apoptosis) and differentiation of a few neurons along the sympathoadrenal cell lineage in the normal development of the sympathetic nervous system. Spontaneous regression is most frequently observed in a subset of disseminated MYCN single-copy neuroblastomas (non-amplified (NA)), termed stage 4s (stage 4s-NA) [10]. However, population-based screening studies for neuroblastomas in Japan, Quebec and Germany suggest that spontaneous regression also occurs in other neuroblastoma subtypes, predominantly localized (stages 1, 2, 3) neuroblastomas (localized-NA) [1113]. Paradoxically, MYCN mRNA and protein levels are higher in favorable localized-NA and, particularly, in stage 4s-NA tumors than in stage 4-NA tumors with poor outcome [14-16], but they do not reach the levels observed in MYCN amplified tumors. In line with this, neuroblastoma cells with elevated MYCN expression retain their capacity to undergo apoptosis [17] or neuronal differentiation [18]. Thus, it has been speculated that MYCN does not only mediate malignant progression in $M Y C N$ amplified tumors, but is also either involved or at least compatible with spontaneous regression in favorable neuroblastomas. In contrast, a functional role of MYCN in stage 4-NA tumors with low MYCN levels is questionable. Here, other transcription factors or pathways within or outside the MYC family of transcription factors could be more relevant. Neuroblastoma-derived cell lines that lack amplified MYCN generally express c-MYC rather than MYCN, often at higher levels than normal tissues [19,20]. However, transcriptional activity of MYCN or c-MYC as reflected by the transcript levels of direct MYCN/c-MYC target genes in relation to $M Y C N$ and c-MYC levels has not yet been defined in neuroblastoma subtypes.

Here, we defined a core set of MYCN and c-MYC target genes by using oligonucleotide microarrays and a neuroblastoma cell line that allows conditional expression of $M Y C N$ or c$M Y C$. Direct regulation of these target genes by $\mathrm{MYCN} / \mathrm{c}-$ MYC was assessed by analyzing the binding of MYCN and cMYC protein to target gene promoters using PCR- and arraybased chromatin immunoprecipitation (ChIP and ChIP-chip, respectively) in different neuroblastoma cell lines. We further investigated the expression of these direct MYCN/c-MYC target genes in relation to $M Y C N$ and c- $M Y C$ expression in different clinical neuroblastoma subtypes. In addition, the association of MYCN/c-MYC target gene expression with overall survival independent of the well-established markers - amplified $M Y C N$, disease stage and age at diagnosis - was demonstrated.

\section{Results \\ Inverse correlation of MYCN and c-MYC expression in neuroblastoma subtypes}

c-MYC mRNA levels are very low in $M Y C N$ amplified tumors (Figure 1), which is due to high MYCN protein repressing c$M Y C$ mRNA expression [20]. Previous quantitative PCR analyses in a cohort of 117 neuroblastoma patients revealed that mRNA levels of $M Y C N$ are significantly lower in stage 4-NA than in stage $4 \mathrm{~s}-\mathrm{NA}(p=0.008)$ and localized-NA neuroblastomas (stages 1, 2, 3; $p=0.03$ ) [14]. To test whether this lower expression of $M Y C N$ in stage 4-NA tumors is due to elevated c-MYC activity that represses $M Y C N$ expression, we analyzed c-MYC and MYCN mRNA levels in a cohort of 251 primary neuroblastoma tumors using a customized $11 \mathrm{~K}$ oligonucleotide microarray (other $M Y C$ gene family members were not differently expressed (data not shown)). Although c-MYC mRNA levels were not significantly higher in stage 4-NA ( $\mathrm{n}=$ $52)$ than in localized-NA tumors $(n=138)$, we found an inverse correlation of $M Y C N$ and c-MYC expression between stage $4 \mathrm{~s}-\mathrm{NA}(\mathrm{n}=30)$ and stage 4 -NA tumors. Stage $4-\mathrm{NA}$ tumors showed lower expression of $M Y C N$ and higher expression of c-MYC, whereas stage $4 \mathrm{~s}-\mathrm{NA}$ tumors showed lower expression of c-MYC and higher expression of $M Y C N$ (Figure $1 ; p=0.008$ for $c-M Y C, p=0.07$ for $M Y C N$ ). 


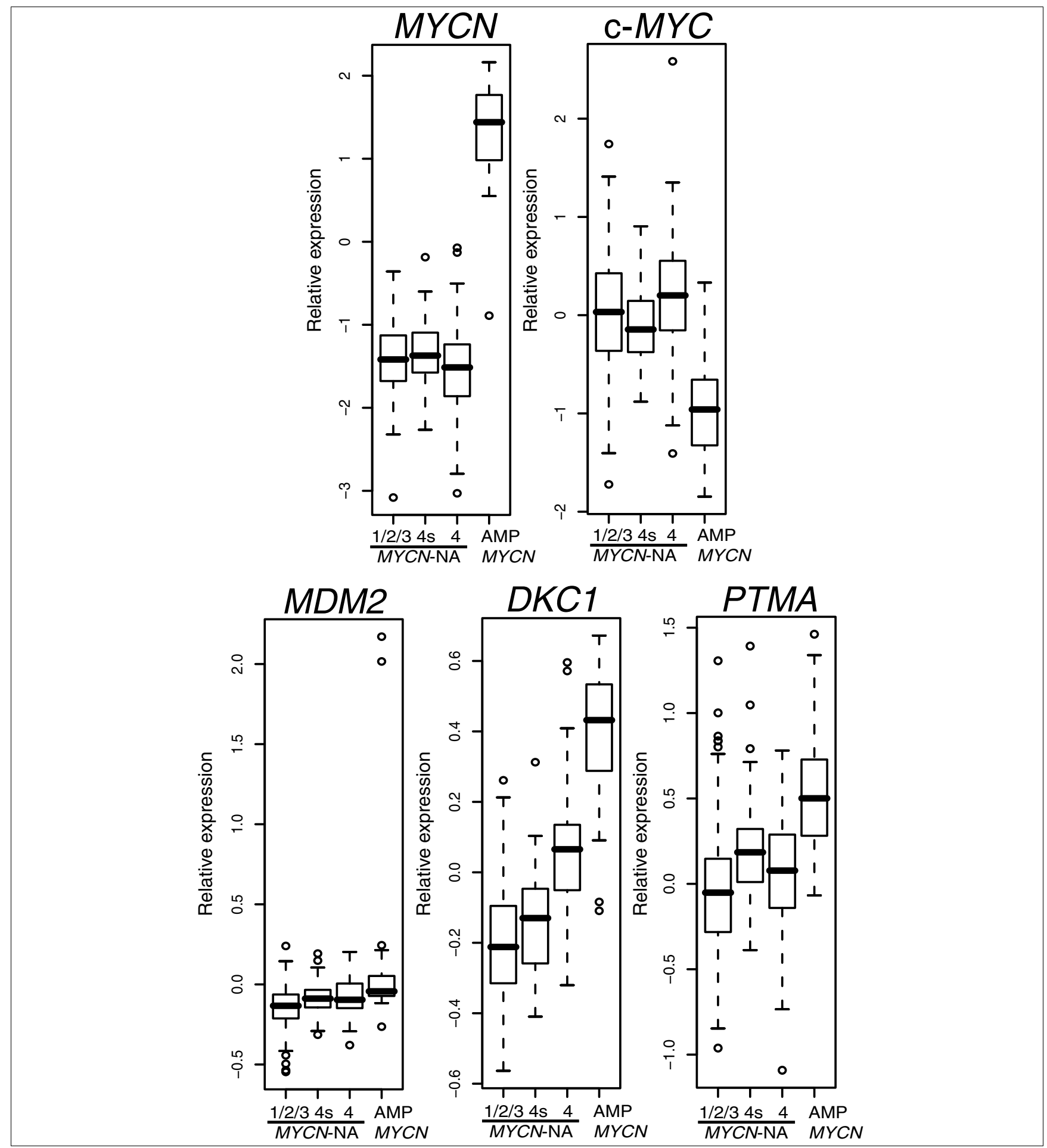

Figure I

Inverse correlation of MYCN and c-MYC mRNA levels in neuroblastoma subtypes. Relative mRNA expression is shown for MYCN and c-MYC as well as for MDM2, DKCI, and PTMA, three direct targets of MYCN/c-MYC. Data are represented as box plots: horizontal boundaries of boxes represent the 25th and 75th percentile. The 50th percentile (median) is denoted by a horizontal line in the box and whiskers above and below extend to the most extreme data point, which is no more than 1.5 times the interquartile range from the box. A set of $25 \mathrm{I}$ primary neuroblastoma tumors was analyzed consisting of I 38 localized-NA (stage I/2/3), 30 stage 4s-NA, 52 stage 4-NA and 3I MYCN amplified (AMP) neuroblastoma tumors. Gene expression levels from stage 4s-NA, stage 4-NA, and MYCN amplified tumors were compared pair-wise with those of localized-NA tumors as reference. Differential gene expression was assessed for each gene by using the Mann-Whitney test (cut-off of $p<0.05$ ). 
Because increased activity of MYCN in stage 4s-NA or c-MYC in stage 4-NA tumors should both result in high expression of shared target genes compared to localized-NA neuroblastomas, we analyzed known direct MYCN/c-MYC target genes, namely $M D M 2$ [21], DKC1 [22], and PTMA [23], in neuroblastoma subtypes. As expected, the highest expression of all three transcripts was observed in $M Y C N$ amplified tumors (Figure $1 ; p<0.001$ for all three transcripts, $\mathrm{n}=31$ ). $M D M 2$ mRNA levels were higher in stage $4-\mathrm{NA}(p=0.005)$ and stage $4 \mathrm{~s}-\mathrm{NA}(p=0.03)$ than in localized-NA tumors (the expression range of $M D M 2$ is large because of two $M Y C N$ amplified tumors with non-syntenic co-amplification of MDM2 (data not shown)). Similarly, $D K C 1$ and $P T M A$ expression was higher in stage 4 -NA $(p<0.001$ for $D K C 1, p=0.02$ for PTMA) and in stage $4 \mathrm{~s}-\mathrm{NA}$ ( $p=0.03$ for $D K C 1, p=0.007$ for PTMA) than in localized-NA tumors. These results suggest an increased MYCN/c-MYC activity also in stage 4S-NA (MYCN) and in stage 4-NA (predominantly c-MYC) compared to localized-NA tumors. However, higher DKC1 mRNA levels in stage 4-NA tumors and higher PTMA mRNA levels in stage 4s-NA tumors also suggest differential regulation of MYCN/c-MYC target genes in these subtypes. To further analyze MYCN/cMYC activity as well as differential regulation of $\mathrm{MYCN} / \mathrm{c}-$ MYC target genes in neuroblastoma subtypes, we thought to define a comprehensive set of target genes directly regulated by MYCN and/or c-MYC in neuroblastoma cells.

\section{Repression of endogenous c-MYC by targeted expression of a MYCN transgene in SH-EPMYCN cells defines C-MYC- and MYCN-regulated genes}

To identify MYCN/c-MYC-regulated genes in neuroblastoma cells, we employed the experimental system SH-EPMYCN, which stably expresses a tetracycline-regulated MYCN transgene [23]. Exponentially growing SH-EPMYCN cells cultured with tetracycline express c-MYC but almost no MYCN protein (Figure 2a). Induction of MYCN by removing tetracycline from the medium is associated with a rapid reduction of cMYC at the mRNA and protein levels. c-MYC reduction occurs prior to the full expression of ectopically induced MYCN protein (Figure 2a). Accordingly, mRNA levels of direct MYCN/c-MYC targets, such as PTMA and DKC1, initially decline before accumulating $\mathrm{MYCN}$ protein leads to the re-induction of these genes. Similar profiles were observed with direct MYCN target genes, such as MDM2 and $M C M 7$ (Additional data file 1).

We used SH-EPMYCN cells for a global search of MYCN and cMYC target genes in neuroblastoma cells using a customized neuroblastoma oligonucleotide microarray (11K, Agilent) that was enriched with probes for genes differentially expressed in neuroblastoma subtypes and for direct MYCN/c-MYC target genes [14,24]. Gene expression profiles of SH-EPMYCN cells at 2, 4, 8, 12, 24, and 48 hours after targeted MYCN expression were generated. Self-organizing maps (SOMs) were used to capture the predominant pattern of gene expression. This analysis yielded 504 clusters (best matching units (BMUs)) consisting, on average, of 20 clones per cluster (Additional data file 1). We searched for clusters with characteristic gene expression profiles of direct MYCN/c-MYC target genes. In addition, known c-MYC target genes from a public database [25] and known MYCN target genes from a literature search were mapped to the 504 clusters (Additional data file 2). A significant enrichment of known MYCN/c-MYC targets was found in 6 clusters (clusters 140, 168, 195, 280, 308, and 336; $p<0.05$, adjusted for multiple testing), consisting of 167 genes. The genes in these six clusters were induced by MYCN and c-MYC in SH-EPMYCN cells. Based on their average gene expression profiles, we grouped the clusters into two subgroups, I and II. Subgroup I genes (clusters 140, 168, and 195) were expressed at equal levels in $\mathrm{SH}-\mathrm{EP}^{M Y C N}$ cells expressing endogenous c-MYC ( 2 hours) and in those fully expressing ectopic MYCN (24 and 48 hours), despite the fact that the maximum protein level of MYCN was significantly higher than that of endogenous c-MYC (Figure 2a; Additional data file 1). This indicates that subgroup I genes are regulated by MYCN, and also suggests that they are less responsive to MYCN than to c-MYC in SH-EPMYCN cells. The mRNA levels of subgroup II genes (clusters 280, 308, and 336) were highest in $\mathrm{SH}-\mathrm{EP}^{M Y C N}$ cells fully expressing ectopic MYCN and followed the combined absolute c-MYC and MYCN protein levels during the time course experiment. We also found clusters with MYCN and c-MYC repressed genes (for example, subgroup III; Additional data file 1). However, enrichment of known MYCN/c-MYC repressed genes from the literature/ database in defined clusters was not found using our statistical cut-off (after adjustment for multiple testing, no cluster showed $p<0.05)$. This was at least partly due to the fact that in SH-EPMYCN cells, some genes were repressed by MYCN but not by c-MYC (subgroup IV). In addition, c-MYC repressed genes from different experimental systems compiled in the cMYC target gene database were not necessarily repressed by MYCN and/or c-MYC in SH-EPMYCN cells.

Therefore, we focused on genes for further validation that were induced by both MYCN and c-MYC proteins in SH$\mathrm{EP}^{M Y C N}$ cells and grouped into subgroup I and II. We extracted all available promoters from the genes represented on the array and scanned for canonical E-boxes (CACGTG) and for the $12 \mathrm{bp} \mathrm{MYCN} \mathrm{position-weight} \mathrm{matrix} \mathrm{[26]} \mathrm{within}$ $-2 \mathrm{~kb}$ and $+2 \mathrm{~kb}$ of the transcriptional start site. We ranked all 504 clusters according to the relative number of putative MYCN/c-MYC binding sites in each cluster. All clusters from subgroups I and II were among the 15 top-ranked clusters with enrichment of predicted MYCN/c-MYC binding sites (data not shown).

To further validate target gene regulation by $\mathrm{MYCN} / \mathrm{c}-\mathrm{MYC}$ in neuroblastoma cells, we performed ChIP-chip using a $244 \mathrm{~K}$ oligonucleotide promoter microarray (Agilent). We analyzed the binding of MYCN and c-MYC to the promoters of the 147 subgroup I and II genes that were represented on the $244 \mathrm{~K}$ promoter microarray. We used five neuroblastoma cell lines 


\section{(a)}
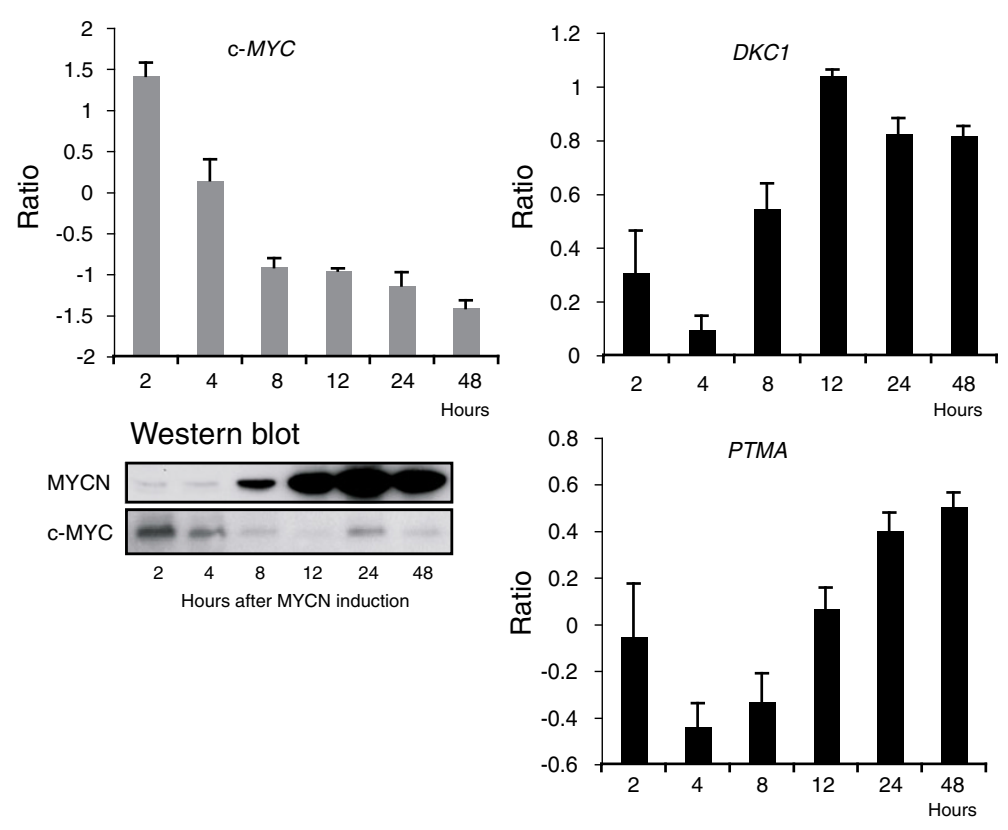

(b)

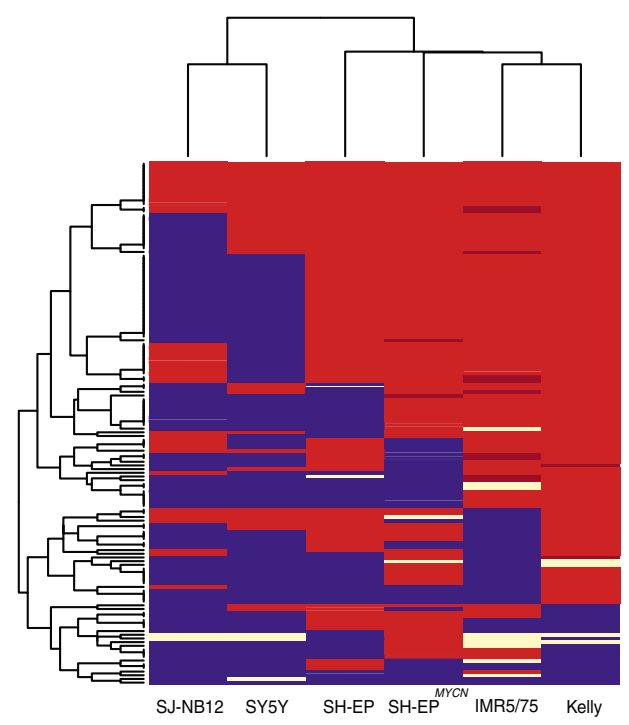

Figure 2

Identification and validation of MYCN/c-MYC target genes in neuroblastoma cell lines. (a) Repression of endogenous c-MYC by targeted expression of a MYCN transgene in SH-EPMYCN cells defines MYCN/c-MYC-regulated genes. MYCN and c-MYC protein levels were monitored in a time series after removing tetracycline in exponentially growing SH-EPMYCN cells that stably express a tetracycline-regulated MYCN transgene. Mean and standard deviation of the relative mRNA levels of MYC, DKCI and PTMA are given from two time series experiments as measured by a customized neuroblastoma oligo microarray. (b) Hierarchical clustering of MYCN- and c-MYC binding to I40 target gene promoters as measured by ChIP-chip in 6 neuroblastoma cell lines. ChIP-chip results of I40 MYCN/c-MYC target genes from 5 neuroblastoma cell lines that preferentially express either high levels of MYCN (SHEPMYCN, IMR5/75 (approximately 75 copies of MYCN) and Kelly (approximately 100-120 copies of MYCN)) or c-MYC (SJ-NBI2 and SY5Y). Additionally, as an intermediate type, parental SH-EP cells were analyzed. SH-EP cells preferentially express C-MYC, but also low levels of MYCN. ChIP-chip experiments were performed with a monoclonal antibody against human MYCN and a polyclonal antibody against human c-MYC for each neuroblastoma cell line. A cut-off for positive binding was set for both transcription factors to $>4$-fold enrichment for one and $>2$-fold enrichment of at least one of the two neighboring probes. MYCN/c-MYC-binding is color-coded as follows: blue, c-MYC binding; red, MYCN/c-MYC binding; dark red, MYCN binding; light yellow, lack of MYCN/c-MYC binding. Hierarchical clustering was used to group neuroblastoma cell lines according to their MYCN/c-MYC-binding pattern. Differentiation between MYCN and c-MYC-binding was mainly achieved through the monoclonal MYCN antibody. The polyclonal antibody against c-MYC also gave positive binding signals for a large set of analyzed target gene promoters in neuroblastoma cell lines with high MYCN that lack cMYC expression (SH-EPMYC, IMR5/75 and Kelly). 
that either preferentially express high levels of MYCN (SHEPMYCN, IMR5/75 (approximately 75 copies of $M Y C N$ ), and Kelly (approximately 100-120 copies of $M Y C N$ )) or c-MYC (SJ-NB12 and $\mathrm{SY}_{5} \mathrm{Y}$ ). Additionally, as an intermediate type, parental SH-EP cells were analyzed, which preferentially express c-MYC, but also MYCN at low level [20,23]. ChIPchip experiments were performed with a monoclonal antibody against human MYCN and a polyclonal antibody against human c-MYC for each of the neuroblastoma cell lines. A cutoff for positive binding was defined as $>4$-fold enrichment for one probe together with $>\mathbf{2}$-fold enrichment for at least one of the two neighboring probes compared to input control. In addition, we manually inspected each of the MYCN and cMYC-binding profiles from the 147 genes. Seven genes were excluded from the analysis because the probe sets for the genes mapped within the genes but outside the target gene promoter regions (all profiles for Kelly and SJ-NB12 cell lines are given in Additional data files 3 and 4, respectively; MYCNand c-MYC-binding results are given in Additional data files 5-7). We also performed PCR-based ChIP for selected candidate genes $(n=13$; Additional data file 8$)$, which all showed analogous results to ChIP-chip (data not shown). Almost all 140 target gene promoters showed binding of MYCN and/or c-MYC in the six analyzed neuroblastoma cell lines as measured by ChIP-chip (Figure 2b). Intriguingly, hierarchical clustering of neuroblastoma cell lines according to the MYCN/cMYC-binding pattern clearly separated MYCN- and c-MYCexpressing neuroblastoma cell lines. Differentiation between MYCN and c-MYC binding was mainly achieved through the monoclonal anti-MYCN antibody. The polyclonal antibody against c-MYC also gave positive binding signals for a large set of target gene promoters in neuroblastoma cell lines with high MYCN that lack detectable c-MYC expression (SHEPMCN, IMR5/75 and Kelly). This was most likely due to unspecific binding of the polyclonal c-MYC antibody to MYCN in these cells. Nevertheless, the lack of binding of MYCN to a large set of target gene promoters in the c-MYCexpressing cells, SJ-NB12 and $\mathrm{SY}_{5} \mathrm{Y}$, and the positive binding of c-MYC to almost all of these target gene promoters in these cells allowed the distinction between MYCN and c-MYC. Taken together, these results indicate that the genes from subgroups I and II represent a core set of target genes directly regulated by either MYCN or c-MYC in neuroblastoma cells dependent on which MYC protein is expressed.

\section{Gradual increase of MYCN/c-MYC target gene expression from stage 4s-NA through stage 4-NA to MYCN amplified tumors}

To determine transcriptional activity of MYCN/c-MYC proteins in primary neuroblastomas $(\mathrm{n}=251)$, we analyzed differential expression of subgroup I and II genes in neuroblastoma subtypes using the Global test as proposed by Goeman et al. [27]. Almost all these genes (154 of 167; 92\%) showed highest expression in MYCN amplified tumors, suggesting that regulation of these genes by MYCN is similar in neuroblastoma cell lines and tumors. Compared to localized-
NA tumors (stages 1, 2, 3), expression of subgroup I and II genes was significantly associated with stage $4 \mathrm{~s}-\mathrm{NA}(p=$ o.002), stage 4-NA $(p<0.001)$ and $M Y C N$ amplified tumors $(p<0.001)$. Global test results further indicated that an increasing number of $\mathrm{MYCN} / \mathrm{c}-\mathrm{MYC}$ target genes was induced from stage 4s-NA through stage 4-NA to $M Y C N$ amplified tumors (Additional data files 9-11). To further illustrate this, we grouped each of the 154 genes into one of four classes based on pair-wise comparisons (Mann-Whitney test, cut-off $p<0.05$ ). These were, compared to localized-NA tumors: overexpressed in $M Y C N$ amplified and in stage 4sNA tumors (class 1); overexpressed in MYCN amplified, stage 4-NA and stage 4s-NA tumors (class 2); overexpressed in $M Y C N$ amplified tumors (class 3); overexpressed in $M Y C N$ amplified and stage 4-NA tumors (class 4) (Figure 3). Compared to localized-NA tumors, 25 (16\%) of the $154 \mathrm{MYCN} / \mathrm{c}-$ MYC target genes, including CCT4, FBL, MDM2, NCL, NPM1, $P T M A$, and TP53, were expressed at higher levels in stage 4sNA tumors (Table 1). Eighty-eight (57\%) of the 154 MYCN/cMYC target genes, including 21 of those overexpressed also in stage 4s-NA tumors, were expressed at higher levels in stage 4-NA than in localized-NA tumors (Table 1, class 2; Additional data file 5). Accordingly, stage 4-NA tumors shared overexpression of 68 of 154 direct MYCN/c-MYC target genes (44\%), including AHCY, RUVBL1, PHB, CDK4, and MRPL3, with $M Y C N$ amplified tumors. Together, this indicates that besides $M Y C N$ amplified tumors, stage 4-NA tumors, and to a lesser extent stage 4s-NA tumors, also show higher MYCN/ c-MYC activity compared to localized-NA tumors. In line with this, we also found lower mRNA levels of an increasing number of MYCN/c-MYC repressed genes from stage 4s-NA (10 out of 68 (15\%) in vitro validated repressed genes that are also lower in $M Y C N$ amplified tumors) through stage 4-NA (34 out of 68 (50\%)) to MYCN amplified tumors (68 out of 102 in vitro validated repressed genes had the lowest expression levels in MYCN amplified tumors (67\%)). Based on the relative expression of $M Y C N$ and $c-M Y C$ in neuroblastoma subtypes, we propose that elevated MYCN activity in stage 4sNA tumors induces only a restricted set of MYCN/c-MYC target genes, whereas elevated c-MYC activity in stage 4-NA tumors induces a larger set of MYCN/c-MYC target genes.

\section{High expression of MYCN/c-MYC target genes is a robust marker of poor overall survival independent of genomic MYCN status, age at diagnosis and disease stage}

Having shown that MYCN/c-MYC target gene activation is also associated with distinct neuroblastoma subtypes, we wanted to test whether MYCN/c-MYC activity as determined by the expression levels of their target genes is associated with overall survival and improves outcome prediction independent of known risk markers. We used the Global test to test the influence of each of the 504 experimentally defined gene clusters on overall survival directly, without the intermediary of single gene testing. The $p$-values for each cluster were adjusted for multiple testing and ranked according to their 
Table I

\begin{tabular}{|c|c|c|c|c|c|c|c|}
\hline Probe & Gene name & Class & BMU & Group & MYCN/c-MYC-fold change* & c-MYC target DB ${ }^{\dagger}$ & Validated by ChIP \\
\hline A_24_P3II604 & C4orf28 & 1 & 195 & 1 & 1.38 & & + \\
\hline A_23_PI02420 & CCT4 & I & 168 & 1 & 1.31 & & + \\
\hline A_23_P555I & $N C L$ & I & 308 & ॥ & 1.69 & Up & + \\
\hline A_23_P44836 & NT5DC2 & I & 140 & 1 & 1.40 & & + \\
\hline A_32_PI39196 & CI3ORF25V_I & 2 & 308 & II & 3.83 & & ND \\
\hline A_24_PI33488 & CDCA4 & 2 & 140 & 1 & 1.45 & & + \\
\hline A_23_PI37I43 & $D K C I$ & 2 & 308 & ॥ & 1.93 & Up & + \\
\hline A_23_P216396 & EXOSC2 & 2 & 308 & II & 1.83 & & + \\
\hline A_23_P78892 & $F B L$ & 2 & 195 & 1 & 1.93 & Up & + \\
\hline A_24_P228796 & GAGE7B & 2 & 195 & 1 & 1.27 & & ND \\
\hline A_23_P4I025 & GNL3 & 2 & 308 & II & 1.80 & $U_{p}$ & ND \\
\hline A_32_P8I 20 & GNL3 & 2 & 308 & II & 1.81 & Up & ND \\
\hline A_23_P398460 & HK2 & 2 & 280 & II & 1.71 & $U_{p}$ & + \\
\hline HsI72673.9 & HsI 72673.9 & 2 & 168 & 1 & 1.73 & & + \\
\hline A_23_P502750 & MDM2 & 2 & 336 & II & 1.19 & ChIP & + \\
\hline A_23_P9226I & MGC2408 & 2 & 280 & II & 2.14 & & + \\
\hline A_23_P50897 & MKI67IP & 2 & 280 & II & 1.97 & $U_{p}$ & + \\
\hline A_23_P2| 4037 & NPMI & 2 & 140 & 1 & 1.61 & $U_{p}$ & + \\
\hline A_23_P57709 & PCOLCE2 & 2 & 308 & II & 2.40 & & + \\
\hline A_24_P34632 & PTMA & 2 & 308 & II & 2.21 & Up & + \\
\hline A_23_PI26825 & SLCI6AI & 2 & 195 & 1 & 1.22 & & + \\
\hline A_23_PI2629I & SNRPE & 2 & 336 & II & 1.49 & & + \\
\hline A_23_PII7068 & SNRPF & 2 & 336 & II & 1.44 & & + \\
\hline A_23_P3I536 & SSBPI & 2 & 336 & II & 1.24 & & + \\
\hline A_23_P26810 & TP53 & 2 & 140 & 1 & 1.44 & $U_{p}$ & + \\
\hline
\end{tabular}

*Fold change expression in SH-EPMYCN cells after MYCN induction. tc-MYC target gene database entry [25]: Up, upregulated; ChIP, validated by ChIP. $\ddagger \bigvee$ alidation of MYCN/c-MYC binding using ChIP in this study (Additional data files 5-7). BMU, best matching unit; ND, not determined.

association with overall survival. Table 2 gives the association with overall survival of the six MYCN/c-MYC target gene clusters and the rank in relation to all other clusters. In a separate analysis, we determined the association with overall survival for each of the 504 experimental gene clusters adjusted for amplified $M Y C N$, stage 4 versus stages 1, 2, 3, and 4s, and age at diagnosis $\geq \mathbf{1} .5$ years (Table 2 ). These well-established risk markers highly correlated with poor outcome in univariate analyses ( $p<0.001$ for each of these three markers). As expected, the Global test without adjustment for co-variables indicated that all MYCN/c-MYC target gene clusters were significantly associated with poor overall survival ( $p<0.001)$. Intriguingly, all six MYCN/c-MYC target gene clusters remained significantly associated with overall survival after adjusting for amplified $M Y C N$, stage 4 versus stages 1, 2, 3, and $4 \mathrm{~s}$, and age at diagnosis $\geq 1.5$ years. Of note, two of the MYCN/c-MYC target gene clusters (clusters 168 and 140, both from subgroup I showing a higher responsiveness to cMYC than to MYCN in SH-EPMYCN) revealed the strongest association with overall survival of all 504 clusters after adjusting for co-variables (Table 2). Figure 4 shows the association with overall survival for each gene from cluster 168 with and without adjustment for co-variables. Most of the genes within this cluster, such as $A H C Y, A R D 1 A, C D K 4$, HSPD1, PHB, RUVBL1, and TRAP1, remained associated with overall survival after adjustment for co-variables. A less significant association with overall survival was observed for clusters with MYCN/c-MYC repressed genes: clusters 454, 482,484 , and 486 were associated with poor overall survival without adjustment for co-variables in the Global test $(p<$ o.001, adjusted for multiple testing), but they showed no significant association with poor overall survival when adjusting for the co-variables amplified $M Y C N$, stage 4 versus stages 1, 2,3 , and $4 \mathrm{~s}$, and age at diagnosis $\geq 1.5$ years. We also asked whether direct MYCN/c-MYC target genes as defined by our analyses are represented in previously published gene expression-based classifiers that distinguish low-risk from high-risk neuroblastomas independent of other risk markers. Gene lists from these studies hardly overlapped, making interpretation difficult. The overlap with our MYCN/c-MYC target gene list was defined by using the gene names as common identifiers. Indeed, different genes defined by our study as direct MYCN/c-MYC target genes were represented in the gene expression classifier gene lists: from the 44 genes over- 


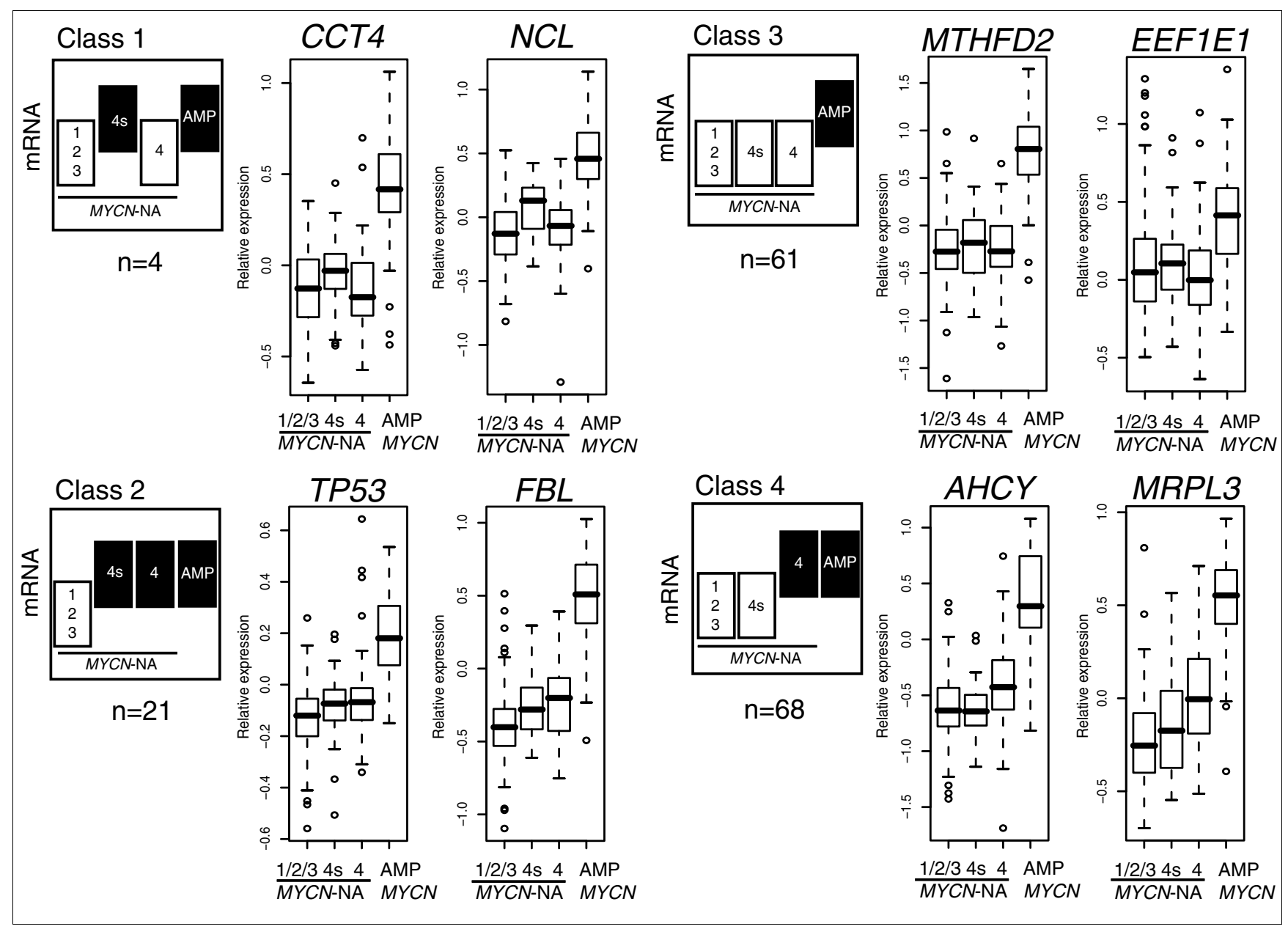

Figure 3

Expression of MYCN/c-MYC target genes in neuroblastoma subtypes. Differential expression was analyzed for each of the genes $(n=154)$ in $M Y C N$ amplified (AMP), stage 4s-NA and stage 4-NA tumors using localized-NA (stage 1/2/3) tumors as reference in pair-wise comparisons (Mann-Whitney test, cut-off $p<0.05$, black). We grouped each of these 154 genes into one of four classes based on their relative expression in clinically relevant neuroblastoma subtypes. These classes were, compared to localized-NA tumors: overexpressed in MYCN amplified and in stage 4s-NA tumors (class I; CCT4 and NCL); overexpressed in MYCN amplified, stage 4-NA and stage 4s-NA tumors (class 2; TP53 and FBL); overexpressed in MYCN amplified tumors (class 3; MTHFD2 and EEFIEI); and overexpressed in MYCN amplified and stage 4-NA tumors (class 4; AHCY and MRPL3).

expressed in high-risk neuroblastomas independent of other markers described by Schramm et al. [28], we identified 10 genes directly regulated by MYCN/c-MYC (DDX21, SCL25A3, EIFA4A2, NME1, NME2, TKT, LDHA, LDHB, HSPD1, $H S P C B$ ); from the 20 genes overexpressed in high-risk neuroblastomas independent of other markers described by Ohira et al. [29], we identified 5 genes directly regulated by MYCN/c-MYC (EEF1G, AHCY, TP53, ENO1, TKT); and from the 66 genes overexpressed in high-risk neuroblastomas independent of other markers described by Oberthuer et al. [24], we identified 7 genes directly regulated by $\mathrm{MYCN} / \mathrm{c}-$ MYC (PRDX4, MRPL3, SNRPE, FBL, LOC2OO916, PAICS, $A H C Y$; Figure 5). Together, these results show that MYCN/cMYC activity as determined by the expression status of a subset of MYCN/c-MYC target genes is significantly associated with poor overall survival independent of other established markers and is a consistent element of gene expression-based neuroblastoma risk classification systems.

\section{Discussion}

In this study, we analyzed MYCN and c-MYC activity as reflected by the expression levels of a core set of direct MYCN/c-MYC targets in neuroblastoma subtypes. As expected, the highest expression levels of MYCN/c-MYC targets were observed in $M Y C N$ amplified tumors. However, we found that besides $M Y C N$ amplified tumors, subtypes of $M Y C N$ single-copy tumors, namely stage 4-NA and, to a lesser extent, stage 4S-NA, also showed increased MYCN/c-MYC target gene activation compared to localized-NA tumors. In general, low MYCN mRNA and protein levels are found in most stage 4-NA tumors [14-16], which does not explain the high mRNA levels of MYCN/c-MYC target genes in this sub- 
Table 2

\begin{tabular}{|c|c|c|c|c|c|}
\hline Cluster & Number of genes & Rank OS* & $p$-value OSt & Rank OS with CV* & $p$-value OS with CVt \\
\hline $168(I)$ & 19 & 3 & $<0.0001$ & 1 & 0.0004 \\
\hline I 40 (I) & 38 & 4 & $<0.0001$ & 2 & 0.0006 \\
\hline 195 (I) & 21 & 31 & $<0.0001$ & 12 & 0.0060 \\
\hline 308 (II) & 33 & 18 & $<0.0001$ & 26 & 0.0161 \\
\hline 280 (II) & 32 & 29 & $<0.0001$ & 37 & 0.0232 \\
\hline 336 (II) & 26 & 51 & $<0.0001$ & 45 & 0.0280 \\
\hline
\end{tabular}

*Rank of all 504 clusters tested for association with overall survival (OS) using the Global test without and with adjustment for co-variables (CV; amplified MYCN, stages I, 2, 3, 4s versus 4 , age at diagnosis $\geq 1.5$ years). tp-value from Global test adjusted for multiple testing. In the Cluster column, I or II gives the cluster group as defined by the SOM analysis using SH-EPMYCN cells.

type. Here, we describe an inverse correlation of $M Y C N$ and $\mathrm{c}-M Y C$ expression levels in stage $4-\mathrm{NA}$ and stage $4 \mathrm{~s}-\mathrm{NA}$ tumors. From experiments in neuroblastoma cell lines, it is known that MYCN and c-MYC control their expression via autoregulatory loops and via repressing each other at defined promoter sites [20]. Neuroblastoma cell lines with high expression of MYCN as a result of amplification lack c-MYC expression. Whenever MYCN and c-MYC are co-expressed in neuroblastoma cell lines, c-MYC expression predominates. Together, this suggests that increased activity of c-MYC represses MYCN in a substantial number of stage 4-NA tumors. In contrast, an inverse regulation, namely the repression of c-MYC by MYCN, is found in $M Y C N$ amplified and, to a lesser extent, in stage 4s-NA tumors. It is important to note that localized-NA tumors also express $M Y C N$ as well as c$M Y C$ and it is likely that they are active because these tumors frequently show high tumor cell proliferation indices [14]. Nevertheless, in localized-NA tumors, we did not observe that one MYC transcription factor dominates over the other, such as in the other neuroblastoma subtypes.

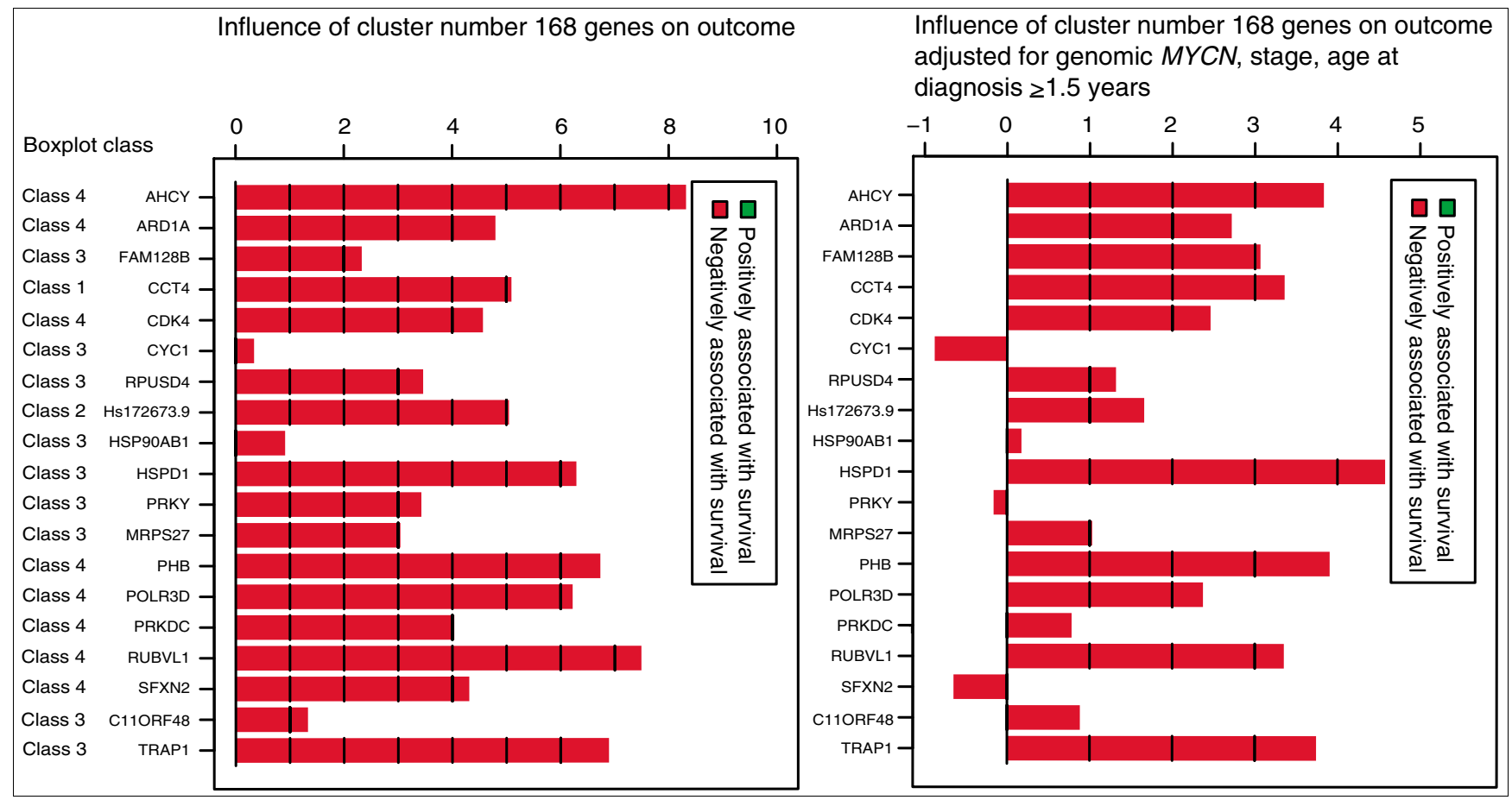

\section{Figure 4}

Association of cluster 168 genes with overall survival. The two gene plots illustrate the influence on overall survival of each gene from cluster 168 . The gene plot gives the influence on overall survival without (left) and with (right) adjustment for the variables genomic MYCN status, age at diagnosis ( $\geq 1.5$ years), and disease stage (stages I, 2, 3, 4s versus stage 4). The gene plot shows a bar and a reference line for each gene tested. In a survival model, the expected height is zero under the null hypothesis that the gene is not associated with the clinical outcome (= reference line). Marks in the bars indicate by how many standard deviations the bar exceeds the reference line. The bars are colored to indicate a negative (red) association of a gene's expression with overall survival. In addition, the boxplot class is given for each gene. 


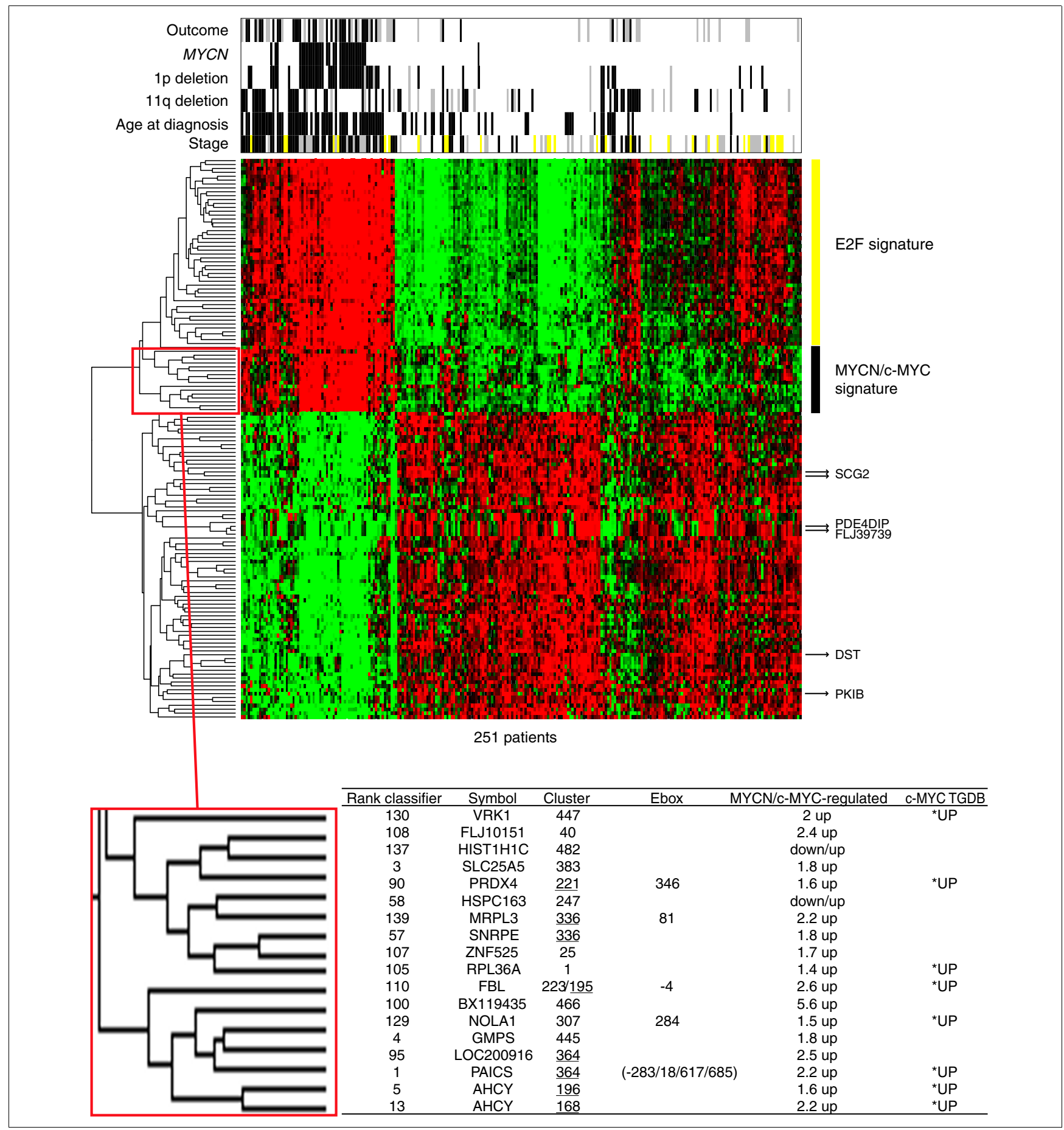

\section{Figure 5}

Representation of MYCN/c-MYC target genes in a gene expression-based neuroblastoma risk stratification system. Two-way hierarchical cluster analysis using 144 oligonucleotide probes from the gene expression-based classifier and the $25 \mathrm{I}$ patients from the entire cohort. Clinical characteristics (outcome, white $=$ no event, gray $=$ relapse/progression, black = death due to neuroblastoma; genomic MYCN status, white = NA, black = amplified; chromosome Ip status, white $=$ normal, black $=$ I $\mathrm{p}$ deleted, gray $=$ not available; chromosome II status, white $=$ normal, black $=$ I Iq deleted, gray $=$ not available; age at diagnosis, white $<I .5$ years, black $\geq I .5$ years; disease stage, white $=$ stage I, 2, gray = stage 3 , yellow $=$ stage $4 \mathrm{~s}$, black $=$ stage 4$)$ are added to the heatmap of gene expression. The gene expression cluster with direct MYCN/c-MYC target genes is highlighted. The Rank Classifier column gives the classifier rank found by the Prediction Analysis for Microarrays algorithm and a complete 10-times-repeated I0-fold cross validation. The Cluster column gives the results from the SOM analysis using gene expression profiles from SH-EPMYCN cells. The MYCN/c-MYC regulated column gives the fold changes after MYCN induction. The Ebox column gives the position of a canonical E-box in the promoter. The c-MYC TGDB column gives the entries in the public cMYC target gene database. *UP, upregulated. 
Our findings further indicate that MYCN/c-MYC target gene activation gradually increases from stage 4S-NA through stage 4-NA to MYCN amplified tumors. High expression of a large number of MYCN/c-MYC target genes was found in stage 4-NA and MYCN amplified tumors, but not in stage $4 \mathrm{~s}^{-}$ NA tumors, which is probably involved in the divergent clinical outcome of these subtypes. This also suggests that MYCN in stage $4 \mathrm{~s}$ tumors is a weaker transactivator than c-MYC in stage 4-NA tumors. Whether this effect is due to the cellular context in which they are expressed and/or due to different functions of the two MYC proteins in neuroblastoma cells is unclear. In favor of a cellular context factor, we observed that promoter constructs from the PTMA gene, which is highly expressed in stage 4S NA and MYCN amplified tumors, showed a strong activation in N-type but not S-type neuroblastoma cell lines despite similar MYCN protein levels (unpublished data). In favor of different functions of the two MYC proteins, our analyses in $\mathrm{SH}-\mathrm{EP}^{M Y C N}$ cells suggest that a large number of MYCN/c-MYC target genes (subgroup I genes) are less responsive to MYCN than to c-MYC. Another unsolved question is which molecular mechanisms induce elevated MYCN activity in stage 4s-NA tumors or elevated cMYC activity in stage 4-NA tumors. Candidate pathways involved in differential regulation of MYC proteins are the Sonic hedgehog pathway (Shh) for MYCN activation [30] and the Wnt/beta-catenin pathway for c-MYC activation [31,32]. However, we observed that $c-M Y C$ mRNA levels are not significantly higher in stage 4-NA than in localized-NA tumors. This suggests that molecular mechanisms that increase c-MYC protein abundance/stability or simply c-MYC activity are involved in MYCN/c-MYC target gene activation in stage 4 tumors.

Our data are in line with a model where stage 4s-NA tumors exhibit a moderate MYCN function gain compared to localized-NA tumors. Both subtypes usually have favorable outcome. Most localized-NA tumors are cured by surgery alone or even regress spontaneously. Stage 4s-NA tumors frequently regress spontaneously but regression can also be induced by a 'mild' chemotherapy. We found that stage 4s-NA tumors express, on average, the highest MYCN mRNA levels of all non-amplified tumors [14]. From the experimentally defined direct MYCN target genes, only a restricted set of 25 genes, including CCT4, FBL, MDM2, NCL, NPM1, PTMA, and $T P_{53}$, was overexpressed in stage 4s-NA compared to localized-NA tumors, indicating that elevated $M Y C N$ in stage $4 \mathrm{~s}^{-}$ NA tumors only partially activates its downstream target genes. On the one hand, this suggests that moderate MYCN function gain in stage 4s-NA tumors is involved in the metastatic phenotype. On the other hand, moderate MYCN function gain in this subtype is still compatible with, or might even favor, spontaneous regression. From the list of MYCN target genes overexpressed in stage 4s-NA tumors, TP53 as a proapoptotic gene, and MDM2, coding for the direct inhibitor of p53 and mediating pro-tumorigenic activities, are strong candidates to be involved in the unique phenotype of stage $4 \mathrm{~s}-\mathrm{NA}$ tumors. However, it is important to note that TP53 and MDM2 are co-expressed at higher levels also in stage 4-NA and $M Y C N$ amplified tumors. Both subtypes initially respond to therapy, but rapidly acquire resistance and frequently show progression/relapse, suggesting that additional conditions activating MDM2 and/or suppressing TP53 functions are acquired. In line with this, alterations disrupting the p14MDM2-p53 pathway, such as MDM2 amplification, p14 methylation/deletion, and TP53 mutations are found in neuroblastoma cell lines that were established from relapsed patients [33]. In this context, it remains to be shown whether small compounds that selectively inhibit MDM2, such as nutlin-3, and that induce proliferation arrest and apoptosis in neuroblastoma cell lines $[34,35]$ represent a new therapeutic option for high-risk neuroblastomas.

\section{Conclusions}

High expression of a defined subset of direct MYCN/c-MYC target genes turned out to be a robust marker for poor overall survival independent of the established markers, amplified $M Y C N$, disease stage (stage 4 versus stages 1, 2, 3, and 4s) and age at diagnosis ( $\geq 1.5$ years). Recently, several gene expression-based neuroblastoma risk stratification systems have been developed that predict outcome more accurately than established risk markers [24,28,29]. Unfortunately, the classifier gene lists emerging from these studies hardly overlap, which has been ascribed to the different composition of the investigated cohorts and the different high-throughput gene expression platforms used. Our data show that markers of increased MYCN/c-MYC activity are consistently represented in these classifier gene lists, indicating that a gene expressionbased classifier that reflects MYCN/c-MYC function should make an attractive tool for neuroblastoma classification and risk prediction.

\section{Materials and methods \\ Patients}

All patients from this study $(\mathrm{n}=251)$ were enrolled in the German Neuroblastoma Trials NB90-NB2004 with informed consent and diagnosed between 1989 and 2004 (patient characteristics are in Additional data files 2 and 12). Tumor samples were collected prior to any cytoreductive treatment. The only criterion for patient selection was availability of sufficient amounts of tumor material. Tumor specimens were checked for at least $60 \%$ tumor content.

\section{Neuroblastoma sample preparation and gene expression analysis}

Gene expression profiles from the tumors were generated as dye-flipped dual-color replicates using customized $11 \mathrm{~K}$ oligonucleotide microarrays as previously described [24]. The $11 \mathrm{~K}$ Agilent microarray was constructed in our laboratory based on extensive neuroblastoma transcriptome information from different whole-genome analyses from primary tumors and 
neuroblastoma cell lines. These also include comparative transcriptome analysis of $M Y C N$ amplified versus not amplified tumors as well as of neuroblastoma cell lines with variable/conditional MYCN/c-MYC expression that allowed the enrichment with MYCN/c-MYC-regulated genes [14,24] (unpublished data). The reference for each tumor RNA was an RNA pool of 100 neuroblastoma tumor samples. Data normalization and quality control is described in Additional data file 2. All raw and normalized microarray data are available at the ArrayExpress database (Accession: E-TABM-38) [36].

Neuroblastoma cell line experiments and SOM analysis The SH-EPMYCN cell line, previously also denoted as TET21N [23], expressing a MYCN transgene under the control of a tetracycline-repressible element was used to generate gene expression profiles from different time points after $M Y C N$ induction showing variable MYCN and c-MYC levels. RNA isolation from $\mathrm{SH}-\mathrm{EP}^{M Y C N}$ cells was performed as previously described [14]. Gene expression profiles were generated as dye-flipped dual-color replicates using the same customized $11 \mathrm{~K}$ oligonucleotide microarray platform used for the tumor samples. The reference for RNA from SH-EPMYCN cells after $M Y C N$ induction was RNA from SH-EPMYCN cells cultured in parallel that lack $M Y C N$ expression. Gene expression profiles from SH-EPMYCN cells with variable MYCN and c-MYC levels were taken for a SOM analysis (Additional data file 2). Protein expression was assessed by immunoblotting using $50 \mu \mathrm{g}$ of total cell lysates from the cell line experiments as previously described [37]. Blots were probed with antibodies directed against MYCN (SantaCruz, sc-53993, Santa Cruz, CA, USA) and c-MYC (SantaCruz, sc-764, Santa Cruz, CA, USA).

\section{ChIP, ChIP-chip and protein analysis}

Chromatin immunoprecipitation was performed as described previously [38,39] using $10 \mu \mathrm{g}$ of MYCN (SantaCruz, sc53993), c-MYC (SantaCruz, sc-764) [40,41] and normal mouse IgG (SantaCruz, sc-2025) antibodies and Dynabeads ProteinG (Invitrogen, Carlsbad, CA, USA). Eluted and purified MYCN-ChIP-DNA ( $1 \mu \mathrm{l})$ of IMR5/75 and $S H-E P M Y C N$ was used as a template in PCR reactions running for 35 cycles. The primer sequences are given in Additional data file 8. In addition, ChIP-DNA templates from $S H-E P^{M Y C N}, S H-E P$, Kelly, $I M R 5 / 75, S J N B-12$ and $S Y_{5} Y$ cells using MYCN and c-MYC antibodies were amplified for DNA microarray analysis (Agilent Human Promoter ChIP-chip Set 244K) using the WGA (Sigma-Aldrich, St. Louis, MO, USA) method [42]. DNA labeling, array hybridization and measurement were performed according to Agilent mammalian ChIP-chip protocols. For the visualization of ChIP-chip results, the cureos package vo.2 for $\mathrm{R}$ was used (available upon request). The in silico promoter analysis for the identification of putative MYC binding sites (canonical and non-canonical E-boxes) is described in Additional data file 2.

\section{Differential gene expression and survival analysis}

Differential gene expression of $M Y C N / c-M Y C$ and their target genes in neuroblastoma tumors was evaluated for stage 4S-NA, stage 4-NA and $M Y C N$ amplified using localized-NA tumors (stages 1, 2, 3) as reference using Goeman's Global test and the Wilcoxon rank sum test. A result was judged as 'statistically significant' at a $p$-value of 0.05 or smaller. Differential expression of $M Y C N$ was evaluated in two partially overlapping cohorts, one measured by quantitative PCR [14] and the other by oligo microarray (the overlap was 101 patients). To test the association of $M Y C N$ in vitro clusters with overall survival (death due to neuroblastoma disease), Goeman's Global test was used [27]. To evaluate the influence of gene expression on outcome independent of established markers, the Global test was adjusted for the following covariables: genomic $M Y C N$ status, stage of the disease (stage 4 versus stages $1,2,3$, and $4 \mathrm{~s}$ ), and age at diagnosis ( $\geq 1.5$ years versus $<1.5$ years). Because of multiple testing of probably dependent gene clusters, $p$-values were adjusted according to Benjamini and Yekutieli [43] to control the false discovery rate of $5 \%$.

\section{Abbreviations}

ChIP, PCR-based chromatin immunoprecipitation; ChIPchip, array-based chromatin immunoprecipitation; NA, nonamplified; SOM, self-organizing map.

\section{Authors' contributions}

FW designed and coordinated the study. FW and DM interpreted results and drafted the manuscript. $\mathrm{AO}, \mathrm{MF}, \mathrm{AB}, \mathrm{BB}$ and FW carried out array-based expression profiling and data analyses of neuroblastoma tumor samples and cell lines. $\mathrm{BH}$ was responsible for clinical data management. TB and RK performed in silico promoter analyses. JV and FP contributed samples and performed literature searches of MYCN/c-MYC target genes. DM performed chromatin immunoprecipitation experiments. DM, TB and FW analyzed ChIP-chip data. AB, $\mathrm{BH}$ and FW carried out global test and survival analyses. FW, DM, KOH, JV, FP and MS contributed to the manuscript. All authors read and approved the final manuscript.

\section{Additional data files}

The following additional data are available. Additional data file 1 is a figure showing a Cluster map of genetic programs regulated by conditional expression of c-MYC and MYCN proteins in SH-EPMYCN cells. Additional data file 2 is a document describing in more detail the methods and materials. Additional data files 3 and 4 are sets of figures showing ChIPchip results of MYCN/c-MYC target genes in the Kelly and SJNB12 cell lines. Additional data files 5, 6 and 7 are tables listing MYCN/c-MYC target genes overexpressed in stage $4 \mathrm{~s}-\mathrm{NA}$, stage 4-NA and MYCN amplified tumors, respectively, compared to localized-NA tumors. Additional data file 8 is a table 
of genes and primers selected to confirm ChIP-chip results. Additional data files 9, 10 and 11 are figures showing the association of MYCN/c-MYC induced genes with neuroblastoma subtypes using the Global test. Additional data file 12 is a table providing patient data.

\section{Acknowledgements}

We thank Steffen Bannert and Yvonne Kahlert for technical assistance. We thank the German Neuroblastoma Tumor Bank for providing tumor samples, the German Neuroblastoma Study Group (study chair Frank Berthold) for providing clinical data and the reference laboratories for providing molecular data. This work was supported by program project grants from the Krebshilfe, BMBF (NGFN2 and Kompetenznetz Pediatric Oncology/Hematology) and the EU. The platform iCHIP (Integration Center of Hlgh throughPut experiments) has been used for the annotation of this study. Jo Vandesompele is a postdoctoral researcher of the Research Foundation - Flanders (FWO-Vlaanderen). Filip Pattyn is supported by a grant of the Ghent University Special Research Fund (BOF).

\section{References}

I. Schwab M, Westermann F, Hero B, Berthold F: Neuroblastoma: biology and molecular and chromosomal pathology. Lancet Oncol 2003, 4:472-480.

2. Vandesompele J, Baudis M, De Preter K, Van Roy N, Ambros P, Bown $\mathrm{N}$, Brinkschmidt $C$, Christiansen $H$, Combaret $V$, Lastowska $M$, Nicholson J, O'Meara A, Plantaz D, Stallings R, Brichard B, Broecke C Van den, De Bie S, De Paepe A, Laureys G, Speleman F: Unequivocal delineation of clinicogenetic subgroups and development of a new model for improved outcome prediction in neuroblastoma. J Clin Oncol 2005, 23:2280-2299.

3. Seeger RC, Brodeur GM, Sather H, Dalton A, Siegel SE, Wong KY, Hammond D: Association of multiple copies of the N-myc oncogene with rapid progression of neuroblastomas. $N$ Engl J Med 1985, 313:1111-1116.

4. Weiss WA, Aldape K, Mohapatra G, Feuerstein BG, Bishop JM: Targeted expression of MYCN causes neuroblastoma in transgenic mice. $E M B O$ J 1997, 16:2985-2995.

5. Schwab M, Varmus HE, Bishop JM: Human N-myc gene contributes to neoplastic transformation of mammalian cells in culture. Nature 1985, 316:160-162.

6. Adhikary S, Eilers M: Transcriptional regulation and transformation by Myc proteins. Nat Rev Mol Cell Biol 2005, 6:635-645.

7. Kleine-Kohlbrecher D, Adhikary S, Eilers M: Mechanisms of transcriptional repression by Myc. Curr Top Microbiol Immunol 2006, 302:5I-62.

8. Prochownik EV, Li Y: The ever expanding role for c-Myc in promoting genomic instability. Cell Cycle 2007, 6:1024-1029.

9. Fulda S, Lutz W, Schwab M, Debatin KM: MycN sensitizes neuroblastoma cells for drug-induced apoptosis. Oncogene 1999, | 8:| |479-| 486.

10. Pritchard J, Hickman JA: Why does stage 4s neuroblastoma regress spontaneously? Lancet 1994, 344:869-870.

II. Sawada T, Hirayama M, Nakata T, Takeda T, Takasugi N, Mori T, Maeda K, Koide R, Hanawa $Y$, Tsunoda A, et al.: Mass screening for neuroblastoma in infants in Japan. Interim report of a mass screening study group. Lancet 1984, 2:27I-273.

12. Woods WG, Tuchman M, Robison LL, Bernstein M, Leclerc JM, Brisson LC, Brossard J, Hill G, Shuster J, Luepker R, Byrne T, Weitzman $S$, Bunin G, Lemieux B: A population-based study of the usefulness of screening for neuroblastoma. Lancet 1996, 348: $1682-1687$.

13. Schilling FH, Spix C, Berthold F, Erttmann R, Sander J, Treuner J, Michaelis J: Children may not benefit from neuroblastoma screening at I year of age. Updated results of the population based controlled trial in Germany. Cancer Lett 2003, 197: 19-28.

14. Westermann F, Henrich KO, Wei JS, Lutz W, Fischer M, Konig R, Wiedemeyer R, Ehemann V, Brors B, Ernestus K, Leuschner I, Benner A, Khan J, Schwab M: High Skp2 expression characterizes highrisk neuroblastomas independent of MYCN status. Clin Cancer Res 2007, 13:4695-4703.

15. Cohn SL, London WB, Huang D, Katzenstein HM, Salwen HR, Rein- hart T, Madafiglio J, Marshall GM, Norris MD, Haber M: MYCN expression is not prognostic of adverse outcome in advanced-stage neuroblastoma with nonamplified MYCN. J Clin Oncol 2000, I 8:3604-36I3.

16. Tang XX, Zhao H, Kung B, Kim DY, Hicks SL, Cohn SL, Cheung NK, Seeger RC, Evans AE, Ikegaki N: The MYCN enigma: significance of MYCN expression in neuroblastoma. Cancer Res 2006, 66:2826-2833.

17. Lutz W, Fulda S, Jeremias I, Debatin KM, Schwab M: MycN and IFNgamma cooperate in apoptosis of human neuroblastoma cells. Oncogene 1998, 17:339-346.

18. Edsjö A, Nilsson H, Vandesompele J, Karlsson J, Pattyn F, Culp LA, Speleman F, Påhlman S: Neuroblastoma cells with overexpressed MYCN retain their capacity to undergo neuronal differentiation. Lab Invest 2004, 84:406-4I7.

19. Sadée W, Yu VC, Richards ML, Preis PN, Schwab MR, Brodsky FM, Biedler JL: Expression of neurotransmitter receptors and myc protooncogenes in subclones of a human neuroblastoma cell line. Cancer Res 1987, 47:5207-52/2.

20. Breit S, Schwab M: Suppression of MYC by high expression of NMYC in human neuroblastoma cells. J Neurosci Res 1989, 24:2I-28.

21. Slack A, Chen Z, Tonelli R, Pule M, Hunt L, Pession A, Shohet JM: The p53 regulatory gene MDM2 is a direct transcriptional target of MYCN in neuroblastoma. Proc Natl Acad Sci USA 2005, 102:73I-736.

22. Boon K, Caron HN, van Asperen R, Valentijn L, Hermus MC, van Sluis P, Roobeek I, Weis I, Voute PA, Schwab M, Versteeg R: N-myc enhances the expression of a large set of genes functioning in ribosome biogenesis and protein synthesis. EMBO J 200I, 20:1383-1393.

23. Lutz W, Stöhr M, Schürmann J, Wenzel A, Löhr A, Schwab M: Conditional expression of $\mathrm{N}$-myc in human neuroblastoma cells increases expression of alpha-prothymosin and ornithine decarboxylase and accelerates progression into S-phase early after mitogenic stimulation of quiescent cells. Oncogene 1996, 13:803-812.

24. Oberthuer A, Berthold F, Warnat P, Hero B, Kahlert Y, Spitz R, Ernestus K, König R, Haas S, Eils R, Schwab M, Brors B, Westermann $F$, Fischer M: Customized oligonucleotide microarray gene expression-based classification of neuroblastoma patients outperforms current clinical risk stratification. I Clin Oncol 2006, 24:5070-5078.

25. Zeller KI, Jegga AG, Aronow BJ, O'Donnell KA, Dang CV: An integrated database of genes responsive to the Myc oncogenic transcription factor: identification of direct genomic targets. Genome Biol 2003, 4:R69.

26. Matys V, Kel-Margoulis OV, Fricke E, Liebich I, Land S, Barre-Dirrie A, Reuter I, Chekmenev D, Krull M, Hornischer K, Voss N, Stegmaier P, Lewicki-Potapov B, Saxel H, Kel AE, Wingender E: TRANSFAC and its module TRANSCompel: transcriptional gene regulation in eukaryotes. Nucleic Acids Res 2006, 34(Database issue):D $108-\mathrm{DI} 10$.

27. Goeman JJ, Oosting J, Cleton-Jansen AM, Anninga JK, van Houwelingen HC: Testing association of a pathway with survival using gene expression data. Bioinformatics 2005, 21:1950-1957.

28. Schramm A, Schulte JH, Klein-Hitpass L, Havers W, Sieverts H, Berwanger B, Christiansen H, Warnat P, Brors B, Eils J, Eils R, Eggert A: Prediction of clinical outcome and biological characterization of neuroblastoma by expression profiling. Oncogene 2005, 24:7902-7912.

29. Ohira M, Oba S, Nakamura Y, Isogai E, Kaneko S, Nakagawa A, Hirata T, Kubo H, Goto T, Yamada S, Yoshida Y, Fuchioka M, Ishii S, Nakagawara $A$ : Expression profiling using a tumor-specific cDNA microarray predicts the prognosis of intermediate risk neuroblastomas. Cancer Cell 2005, 7:337-350.

30. Hatton BA, Knoepfler PS, Kenney AM, Rowitch DH, de Alboran IM, Olson JM, Eisenman RN: $\mathbf{N}$-myc is an essential downstream effector of Shh signaling during both normal and neoplastic cerebellar growth. Cancer Res 2006, 66:8655-866I.

31. Liu X, Mazanek P, Dam V, Wang Q, Zhao H, Guo R, Jagannathan J, Cnaan A, Maris JM, Hogarty MD: Deregulated Wnt/beta-catenin program in high-risk neuroblastomas without MYCN amplification. Oncogene 2008, 27:1478-1488.

32. van de Wetering M, Sancho E, Verweij C, de Lau W, Oving I, Hurlstone A, van der Horn K, Batlle E, Coudreuse D, Haramis AP, TjonPon-Fong M, Moerer P, van den Born M, Soete G, Pals S, Eilers M, Medema R, Clevers H: The beta-catenin/TCF-4 complex 
imposes a crypt progenitor phenotype on colorectal cancer cells. Cell 2002, I I I :24 I-250.

33. Carr J, Bell E, Pearson AD, Kees UR, Beris H, Lunec J, Tweddle DA: Increased frequency of aberrations in the p53/MDM2/ p I 4(ARF) pathway in neuroblastoma cell lines established at relapse. Cancer Res 2006, 66:2138-2I45.

34. Van Maerken T, Speleman F, Vermeulen J, Lambertz I, De Clercq S, De Smet E, Yigit N, Coppens V, Philippé J, De Paepe A, Marine JC, Vandesompele ]: Small-molecule MDM2 antagonists as a new therapy concept for neuroblastoma. Cancer Res 2006, 66:9646-9655.

35. Barbieri E, Mehta P, Chen Z, Zhang L, Slack A, Berg S, Shohet JM: MDM2 inhibition sensitizes neuroblastoma to chemotherapy-induced apoptotic cell death. Mol Cancer Ther 2006, 5:2358-2365.

36. Brazma A, Parkinson $H$, Sarkans U, Shojatalab M, Vilo J, Abeygunawardena N, Holloway E, Kapushesky M, Kemmeren P, Lara GG, Oezcimen A, Rocca-Serra P, Sansone SA: ArrayExpress-a public repository for microarray gene expression data at the EBI. Nucleic Acids Res 2003, 3 I:68-7I.

37. Wiedemeyer R, Westermann F, Wittke I, Nowock J, Schwab M: Ataxin-2 promotes apoptosis of human neuroblastoma cells. Oncogene 2003, 22:40I-4II.

38. Strieder V, Lutz W: E2F proteins regulate MYCN expression in neuroblastomas. J Biol Chem 2003, 278:2983-2989.

39. Lee TI, Johnstone SE, Young RA: Chromatin immunoprecipitation and microarray-based analysis of protein location. Nat Protoc 2006, I:729-748.

40. Knoepfler PS, Zhang XY, Cheng PF, Gafken PR, McMahon SB, Eisenman RN: Myc influences global chromatin structure. EMBO J 2006, 25:2723-2734.

4I. Guccione E, Martinato F, Finocchiaro G, Luzi L, Tizzoni L, Dall' Olio V, Zardo G, Nervi C, Bernard L, Amati B: Myc-binding-site recognition in the human genome is determined by chromatin context. Nat Cell Biol 2006, 8:764-770.

42. O'Geen H, Nicolet CM, Blahnik K, Green R, Farnham PJ: Comparison of sample preparation methods for ChIP-chip assays. Biotechniques 2006, 41:577-580.

43. Benjamini $Y$, Yekutieli D: The control of the false discovery rate in multiple testing under dependency. Ann Stat 200I, 29: I 165-I I88.

44. Brodeur GM, Pritchard J, Berthold F, Carlsen NL, Castel V, Castelberry RP, De Bernardi B, Evans AE, Favrot M, Hedborg F, et al.: Revisions of the international criteria for neuroblastoma diagnosis, staging, and response to treatment. J Clin Oncol 1993, I I:| 466-1477.

45. Ambros PF, Ambros IM, SIOP Europe Neuroblastoma Pathology, Biology, and Bone Marrow Group: Pathology and biology guidelines for resectable and unresectable neuroblastic tumors and bone marrow examination guidelines. Med Pediatr Oncol 200I, 37:492-504.

46. CRAN [http://www.r-project.org]

47. Bioconductor [http://www.bioconductor.org]

48. Buness A, Huber W, Steiner K, Sultmann H, Poustka A: arrayMagic: two-colour cDNA microarray quality control and preprocessing. Bioinformatics 2005, 2 I:554-556.

49. Huber W, von Heydebreck A, Sültmann H, Poustka A, Vingron M: Variance stabilization applied to microarray data calibration and to the quantification of differential expression. Bioinformatics 2002, I 8(SuppI I):S96-SI04.

50. Hubbard TJ, Aken BL, Beal K, Ballester B, Caccamo M, Chen Y, Clarke L, Coates G, Cunningham F, Cutts T, Down T, Dyer SC, Fitzgerald S, Fernandez-Banet J, Graf S, Haider S, Hammond M, Herrero J, Holland R, Howe K, Howe K, Johnson N, Kahari A, Keefe D, Kokocinski F, Kulesha E, Lawson D, Longden I, Melsopp C, Megy K, et al:: Ensembl 2007. Nucleic Acids Res 2007, 35(Database issue):D610-D617.

51. MYCNot [http://medgen.ugent.be/MYCNot/]

52. Kohonen T: Self-organizing Maps Second edition. Heidelberg: Springer; 1997. [Springer Series in Information Sciences, volume 30]

53. SOM Toolbox [http://www.cis.hut.fi/projects/somtoolbox/] 